\title{
Normal Matrices in Degenerate Indefinite Inner Product Spaces
}

\author{
Christian Mehl* Carsten Trunk*
}

October 25, 2006

\begin{abstract}
Complex matrices that are structured with respect to a possibly degenerate indefinite inner product are studied. Based on the theory of linear relations, the notion of an adjoint is introduced: the adjoint of a matrix is defined as a linear relation which is a matrix if and only if the inner product is nondegenerate. This notion is then used to give alternative definitions of selfadjoint and unitary matrices in degenerate inner product spaces and it is shown that those coincide with the definitions that have been used in the literature before. Finally, a new definition for normal matrices is given which allows the generalization of an extension result for positive invariant subspaces from the case of nondegenerate inner products to the case of degenerate inner products.
\end{abstract}

\section{Introduction}

We consider the space $\mathbb{C}^{n}$ equipped with an indefinite inner product induced by a Hermitian matrix $H \in \mathbb{C}^{n \times n}$ via

$$
[x, y]:=[x, y]_{H}:=\langle H x, y\rangle=y^{*} H x
$$

where $\langle\cdot, \cdot\rangle$ denotes the standard Euclidean scalar product on $\mathbb{C}^{n}$. We will suppress the subscript $H$ when it is clear that $H$ induces the indefinite inner product.

If $H$ is invertible, i.e., if the indefinite inner product is nondegenerate, then for a matrix $M \in \mathbb{C}^{n \times n}$ there exists a unique matrix $M^{[*]_{H}}$ satisfying

$$
[x, M y]=\left[M^{[*]_{H}} x, y\right] \quad \text { for all } x, y \in \mathbb{C}^{n} .
$$

(Again, we will suppress the subscript $H$ if it is clear from the context which inner product is under consideration.) This matrix $M^{[*]}$ is called the $H$-adjoint of $M$. From (1.2), one easily obtains the matrix identity

$$
M^{[*]}=H^{-1} M^{*} H .
$$

*Technische Universität Berlin, Institut für Mathematik, Sekretariat MA 4-5, D-10623 Berlin, Germany, (mehl@math.tu-berlin.de, trunk@math.tu-berlin.de 
A matrix $M \in \mathbb{C}^{n \times n}$ is called $H$-selfadjoint, $H$-skewadjoint, or $H$-unitary, respectively, if $M^{[*]}=M, M^{[*]}=-M$, or $M^{[*]}=M^{-1}$, respectively. Using (1.3), we obtain the matrix identities

$$
M^{*} H=H M, \quad M^{*} H+H M=0, \quad \text { or } \quad M^{*} H M=H
$$

for $H$-selfadjoint, $H$-skewadjoint, or $H$-unitary matrices, respectively. These three types of matrices have been widely discussed in the literature, both in terms of theory and numerical analysis. Extensive lists of references can be found in $[1,9,13,21]$.

$H$-selfadjoint, $H$-skewadjoint, and $H$-unitary matrices are special cases of $H$-normal matrices. A matrix $M \in \mathbb{C}^{n \times n}$ is called $H$-normal if $M$ commutes with its $H$-adjoint, i.e., if $M M^{[*]}=M^{[*]} M$, or, in other words, if and only if

$$
M H^{-1} M^{*} H=H^{-1} M^{*} H M, \quad \text { or, } \quad H M H^{-1} M^{*} H=M^{*} H M .
$$

In recent years, there has been great interest in $H$-normal matrices, see $[8,9,10,17,18]$ and the references therein.

Spaces with degenerate inner products, that is, $H$ is singular, are less familiar, although this case does appear in applications [14]. Some works here, primarily concerning infinite dimensional degenerate Pontryagin spaces, include [23], [11] (and references there), [2], [12], and parts of the book [3]. The main problem in the context of degenerate inner products is that there is no straightforward definition of an $H$-adjoint. Indeed, if $H$ is singular, then for a matrix $M \in \mathbb{C}^{n \times n}$ an $H$-adjoint, i.e., a matrix $N \in \mathbb{C}^{n \times n}$ satisfying $[x, M y]=[N x, y]$ for all $x, y \in \mathbb{C}^{n}$ need not exist, and if it exists, it need not be unique.

Example 1.1 Let $a, b \in \mathbb{C}$ and

$$
H=\left(\begin{array}{ll}
1 & 0 \\
0 & 0
\end{array}\right), \quad M_{1}=\left(\begin{array}{ll}
1 & 0 \\
0 & 1
\end{array}\right), \quad M_{2}=\left(\begin{array}{ll}
1 & 1 \\
0 & 1
\end{array}\right), \quad N(a, b)=\left(\begin{array}{ll}
1 & 0 \\
a & b
\end{array}\right) .
$$

Then for all possible choices $a, b \in \mathbb{C}, N(a, b)$ satisfies $\left[x, M_{1} y\right]=[N(a, b) x, y]$ for all $x, y \in \mathbb{C}^{n}$. On the other hand, there is no matrix $N \in \mathbb{C}^{2 \times 2}$ such that $\left[x, M_{2} y\right]=[N x, y]$ for all $x, y \in \mathbb{C}^{n}$.

Despite the lack of the notion of an adjoint, $H$-selfadjoint, $H$-skewadjoint and $H$-unitary for the case of singular $H$ can be defined by the matrix identities (1.4) and this definition has been used in many sources, see, e.g., [20] and the references therein. The corresponding matrix identities (1.5) for $H$-normal matrices, however, require an inverse of $H$. A standard approach to circumvent this difficulty is the use of some generalized inverse, in particular the Moore-Penrose generalized inverse $H^{\dagger}$, instead. However, it seems that the use of the Moore-Penrose inverse leads to some inconsistencies in the theory of degenerate inner products. We illustrate this by help of an example.

Example 1.2 Let

$$
H=\left(\begin{array}{ll}
1 & 0 \\
0 & 0
\end{array}\right), \quad A=\left(\begin{array}{ll}
1 & 0 \\
1 & 1
\end{array}\right), \quad N=\left(\begin{array}{ll}
0 & 1 \\
1 & 0
\end{array}\right) .
$$


Then we have $H^{\dagger}=H$. Observe that $A^{*} H=H A$, but $A \neq H^{\dagger} A^{*} H$. So, should $A$ be considered as an $H$-selfadjoint matrix or not? On the other hand, note that $N H^{\dagger} N^{*} H=$ $H^{\dagger} N^{*} H N$, but $H N H^{\dagger} N^{*} H \neq N^{*} H N$. So, should $N$ be considered as an $H$-normal matrix or not?

In [16] $H$-normal matrices have been defined as matrices $M$ satisfying

$$
H M H^{\dagger} M^{*} H=M^{*} H M
$$

and this definition has later been taken up in $[4,17]$. (This way minimizes the number of times the Moore-Penrose generalized inverse of $H$ appears in the defining equation of $H$-normal matrices.) In this paper, we will call matrices $M$ satisfying (1.6) Moore-Penrose $H$-normal matrices in order to highlight the occurrence of the Moore-Penrose generalized inverse in the definition.

Although it is possible to prove some interesting results for Moore-Penrose $H$-normal matrices (e.g., concerning existence of invariant maximal semidefinite subspaces), there is an unpleasant mismatch between $H$-selfadjoint, $H$-skewadjoint, and $H$-unitary matrices on the one hand and Moore-Penrose $H$-normal matrices on the other hand. It is easy to check (see also [20]) that the kernel of $H$ is always an invariant subspace for $H$-selfadjoint, $H$-skewadjoint, and $H$-unitary matrices (defined as in (1.4)). However, it has been shown in [17, Example 6.1] that there exist Moore-Penrose $H$-normal matrices $A$ such that ker $H$ is not $A$-invariant.

It is exactly the fact that ker $H$ need not be invariant which makes the investigation of Moore-Penrose $H$-normal matrices challenging. For example, let us consider the problem of existence of semidefinite invariant subspaces. Recall that a subspace $\mathcal{M} \subseteq \mathbb{C}^{n}$ is called $H$-nonnegative if if $[x, x] \geq 0$ for every $x \in \mathcal{M}, H$-positive if $[x, x]>0$ for every nonzero $x \in \mathcal{M}$, and $H$-neutral if $[x, x]=0$ for every $x \in \mathcal{M}$. An $H$-nonnegative subspace is said to be maximal $H$-nonnegative if it is not properly contained in any larger $H$-nonnegative subspace. It is easy to see that an $H$-nonnegative subspace is maximal if and only if its dimension is equal to $\nu_{+}(H)+\nu_{0}(H)$, where $\nu_{+}(H)$ and $\nu_{0}(H)$ denote the the number (counted with multiplicities) of positive and zero eigenvalues of $H$, respectively. It has been shown in [17, Theorem 6.6] that any Moore-Penrose $H$-normal matrix has a maximal $H$-nonnegative invariant subspace, but the problem which $H$-nonnegative, $H$-positive, or $H$-neutral invariant subspaces of Moore-Penrose $H$-normal matrices can be extended to a maximal $H$-nonnegative invariant subspaces has only been solved for the case of invertible $H$ so far $[18,19]$.

It is the aim of this paper to propose a different definition of $H$-normal matrices in degenerate inner product spaces that is based on a generalization of the $H$-adjoint $A^{[*]_{H}}$ of a matrix $A$ for singular $H$. This generalization is obtained by dropping the assumption that the $H$-adjoint of a matrix is a matrix itself. Instead, the $H$-adjoint $A^{[*]_{H}}$ is defined to be a linear relation in $\mathbb{C}^{n}$, i.e., a linear subspace of $\mathbb{C}^{2 n}$. For basic facts on linear relations and further references see, e.g., [5, 6, 7, 22]. Throughout this paper we identify a matrix $A \in \mathbb{C}^{n \times n}$ with its graph

$$
\left\{\left(\begin{array}{c}
x \\
A x
\end{array}\right): x \in \mathbb{C}^{n}\right\} \subseteq \mathbb{C}^{2 n}
$$


which is a linear relation, also denoted by $A$. If $H \in \mathbb{C}^{n \times n}$ is invertible, then, by (1.2), we obtain that $A^{[*]_{H}}$ coincides with the linear relation

$$
A^{[*]_{H}}=\left\{\left(\begin{array}{c}
y \\
z
\end{array}\right) \in \mathbb{C}^{2 n}:[y, A x]_{H}=[z, x]_{H} \text { for all } x \in \mathbb{C}^{n}\right\} .
$$

This representation allows a direct generalization of the concept of adjoint to the case of degenerate inner products and even to the case of starting with a linear relation rather than a matrix $A$, see [22].

Definition 1.3 Let $H \in \mathbb{C}^{n \times n}$ be Hermitian and let $A$ be a linear relation in $\mathbb{C}^{n}$. Then the linear relation

$$
A^{[*]_{H}}=\left\{\left(\begin{array}{l}
y \\
z
\end{array}\right) \in \mathbb{C}^{2 n}:[y, w]_{H}=[z, x]_{H} \text { for all }\left(\begin{array}{c}
x \\
w
\end{array}\right) \in A\right\}
$$

is called the $H$-adjoint of $A$.

Again, if there is no risk of ambiguity, we will suppress the subscript $H$ in the notation. In this setting, we obtain (see Proposition 2.6 below) that

$$
A^{[*]}=H^{-1} A^{*} H
$$

where $H^{-1}$ is the inverse of $H$ in the sense of linear relations (see Section 2). Observe that this coincides with (1.3) if $H$ is invertible. Hence, the $H$-adjoint in degenerate inner product spaces is a natural generalization of the $H$-adjoint in nondegenerate inner product spaces.

The remainder of the paper is organized as follows. In Section 2, we discuss basic properties of the $H$-adjoint. In Section 3 we use the $H$-adjoint to define $H$-symmetric and $H$-isometric linear relations and we show that in the case of matrices these definitions coincide with the definitions of $H$-selfadjoint and $H$-unitary matrices via the identities (1.4). In Section 4, we present a new definition for $H$-normal matrices. We show that the set of $H$-normal matrices is a proper subset of the set of Moore-Penrose $H$-normal matrices and that $H$-normal matrices share the property with $H$-selfadjoint and $H$-unitary matrices that the kernel of $H$ is always an invariant subspace. The latter fact allows us to obtain sufficient conditions for an $H$-positive invariant subspace of an $H$-normal matrix to be contained in a maximal $H$-nonnegative invariant subspace. This generalizes a result obtained in $[18]$.

\section{The adjoint in degenerate inner product spaces}

We study linear relations in $\mathbb{C}^{n}$, i.e., linear subspaces of $\mathbb{C}^{2 n}$. For the definitions of linear operations with relations and the inverse of relations we refer to [6]. We only mention the 
following. For linear relations $A, B \subseteq \mathbb{C}^{2 n}$ we define

$$
\begin{array}{ll}
\operatorname{dom} A=\left\{x:\left(\begin{array}{l}
x \\
y
\end{array}\right) \in A\right\}, & \text { the domain of } A, \\
\operatorname{ran} A=\left\{y:\left(\begin{array}{l}
x \\
y
\end{array}\right) \in A\right\}, & \text { the range of } A, \\
\operatorname{mul} A=\left\{y:\left(\begin{array}{l}
0 \\
y
\end{array}\right) \in A\right\}, & \text { the multivalued part of } A, \\
A^{-1}=\left\{\left(\begin{array}{l}
y \\
x
\end{array}\right):\left(\begin{array}{l}
x \\
y
\end{array}\right) \in A\right\}, & \text { the inverse of } A
\end{array}
$$

and the product of $A$ and $B$,

$$
A B=\left\{\left(\begin{array}{l}
x \\
z
\end{array}\right): \text { there exists a } y \in \mathbb{C}^{n} \text { with }\left(\begin{array}{l}
y \\
z
\end{array}\right) \in A,\left(\begin{array}{l}
x \\
y
\end{array}\right) \in B\right\} .
$$

In all cases, $x, y, z$ are understood to be from $\mathbb{C}^{n}$. For a subset $M \subseteq \mathbb{C}^{n}$ we define

$$
M^{[\perp]}=\{x:[x, y]=0 \text { for all } y \in M\} .
$$

The following lemma is needed for the proof of Proposition 2.2 below. It is contained in [15, proof of Lemma 2.2], but for the sake of completeness we give a separate proof.

Lemma 2.1 Let $M \subseteq \mathbb{C}^{n}$ be a subspace. Then $\left(M^{[\perp]}\right)^{[\perp]}=M+\operatorname{ker} H$.

Proof. Obviously, we have $M+\operatorname{ker} H \subseteq\left(M^{[\perp]}\right)^{[\perp]}$. If $u \notin M+\operatorname{ker} H$ then, as the quotient space $\left(\mathbb{C}^{n} / \operatorname{ker} H,[\cdot, \cdot]^{\sim}\right)$ with $[x+\operatorname{ker} H, y+\operatorname{ker} H]^{\sim}:=[x, y], x, y \in \mathbb{C}^{n}$, is nondegenerate, there exists an $v \in \mathbb{C}^{n}$ such that $[v, M]=[v+\operatorname{ker} H, M+\operatorname{ker} H]^{\sim}=\{0\}$ and $[v, u]=$ $[v+\operatorname{ker} H, u+\operatorname{ker} H]^{\sim} \neq 0$. Therefore $u \notin\left(M^{[\perp]}\right)^{[\perp]}$, which completes the proof.

In the next proposition we collect some properties of the $H$-adjoint.

Proposition 2.2 Let $A, B \subseteq \mathbb{C}^{2 n}$ be linear relations. Then we have

(i) $A \subseteq B \quad \Longrightarrow \quad B^{[*]} \subseteq A^{[*]}$.

(ii) $\operatorname{mul} A^{[*]}=(\operatorname{dom} A)^{[\perp]}$. If $A$ is a matrix, we have mul $A^{[*]}=\operatorname{ker} H$.

(iii) $\operatorname{ker} A^{[*]}=(\operatorname{ran} A)^{[\perp]}$.

(iv) $\left(A^{[*]}\right)^{[*]}=A+(\operatorname{ker} H \times \operatorname{ker} H)$.

Proof. Assertions (i), (ii), (iii) are easy consequences of Definition 1.3 and can be found, e.g., in $[22]$. 
In order to prove assertion (iv) we equip the space $\mathbb{C}^{n} \times \mathbb{C}^{n}$ with the inner product

$$
\llbracket\left(\begin{array}{l}
y \\
z
\end{array}\right),\left(\begin{array}{l}
x \\
w
\end{array}\right) \rrbracket:=i([y, w]-[z, x])=\left\langle\left(\begin{array}{cc}
0 & -i H \\
i H & 0
\end{array}\right)\left(\begin{array}{l}
y \\
z
\end{array}\right),\left(\begin{array}{l}
x \\
w
\end{array}\right)\right\rangle
$$

where $x, y, w, z \in \mathbb{C}^{n}$. Then

$$
A^{[*]}=A^{\llbracket \perp \rrbracket} \quad \text { and }\left(A^{[*]}\right)^{[*]}=\left(A^{\llbracket \perp \rrbracket}\right)^{\llbracket \perp \rrbracket} .
$$

Lemma 2.1 remains true if we replace $\mathbb{C}^{n}$ by $\mathbb{C}^{2 n},[\perp]$ by $\llbracket \perp \rrbracket$ and $\operatorname{ker} H$ by $(\operatorname{ker} H \times \operatorname{ker} H)$. This and (2.1) shows assertion (iv) of Proposition 2.2.

If $A$ is a matrix, then, by Proposition 2.2 (iv), we have that $y \in \operatorname{mul}\left(A^{[*]}\right)^{[*]}$ if and only if there exists $f, g \in \operatorname{ker} H$ such that

$$
\left(\begin{array}{l}
0 \\
y
\end{array}\right)=\left(\begin{array}{c}
f \\
A f
\end{array}\right)+\left(\begin{array}{c}
-f \\
g
\end{array}\right)=\left(\begin{array}{c}
0 \\
A f+g
\end{array}\right) .
$$

This proves the following lemma.

Lemma 2.3 Let $A \in \mathbb{C}^{n \times n}$ be a matrix. Then $\operatorname{ker} H \subseteq \operatorname{mul}\left(A^{[*]}\right)^{[*]}$. If , in addition, ker $H$ is A-invariant, then we have

$$
\operatorname{mul}\left(A^{[*]}\right)^{[*]}=\operatorname{ker} H .
$$

The following formula for the $H$-adjoint of matrices will be used frequently.

Lemma 2.4 Let $A \in \mathbb{C}^{n \times n}$ be a matrix. Then

$$
A^{[*]}=\left\{\left(\begin{array}{l}
y \\
z
\end{array}\right) \in \mathbb{C}^{2 n}: A^{*} H y=H z\right\} .
$$

In particular, $A^{[*]}$ is a matrix if and only if $H$ is invertible.

Proof. Clearly, since $A$ is a matrix, we have

$$
\left(\begin{array}{l}
y \\
z
\end{array}\right) \in A^{[*]} \Longleftrightarrow x^{*} A^{*} H y=x^{*} H z \text { for all } x \in \mathbb{C}^{n} .
$$

This implies (2.2). The remaining assertion of Lemma 2.4 follows from Proposition 2.2 (ii).

By Lemma 2.4 we have that $\operatorname{dom} A^{[*]}=\mathbb{C}^{n}$ if and only if $\operatorname{ran}\left(A^{*} H\right) \subseteq \operatorname{ran} H$.

Example 2.5 Using the notations of Example 1.1 we have

$$
M_{1}^{[*]}=\operatorname{graph} I+(\{0\} \times\{0\} \times\{0\} \times \mathbb{C}) \text { and } M_{2}^{[*]}=\{0\} \times \mathbb{C} \times\{0\} \times \mathbb{C},
$$

where + denotes the sum of linear subspaces. We then obtain

$$
\begin{array}{rlrl}
\operatorname{dom} M_{1}^{[*]} & =\mathbb{C}^{2}, & & \operatorname{mul} M_{1}^{[*]}=\{0\} \times \mathbb{C}, \\
\operatorname{dom} M_{2}^{[*]}=\{0\} \times \mathbb{C}, & \operatorname{mul} M_{2}^{[*]}=\{0\} \times \mathbb{C} .
\end{array}
$$


Let $A \subseteq \mathbb{C}^{2 n}$ be a linear relation. Introducing a change of basis $x \mapsto P x$ on $\mathbb{C}^{n}$, where $P \in \mathbb{C}^{n \times n}$ is a nonsingular matrix, yields

$$
P^{-1} A P=\left\{\left(\begin{array}{c}
P^{-1} x \\
P^{-1} w
\end{array}\right):\left(\begin{array}{c}
x \\
w
\end{array}\right) \in A\right\} .
$$

Therefore, for

$$
\left(\begin{array}{c}
y \\
z
\end{array}\right) \in\left(P^{-1} A P\right)^{[*]_{P^{*} H}} \quad \text { and arbitrary }\left(\begin{array}{c}
x \\
w
\end{array}\right) \in A
$$

we have $0=\left[y, P^{-1} w\right]_{P^{*} H P}-\left[z, P^{-1} x\right]_{P^{*} H P}=[P y, w]_{H}-[P z, x]_{H}$, i.e.,

$$
\left(\begin{array}{c}
P y \\
P z
\end{array}\right) \in A^{[*]_{H}} \quad \text { or, equivalently, }\left(\begin{array}{c}
y \\
z
\end{array}\right) \in P^{-1} A^{[*]_{H}} P .
$$

This gives

$$
\left(P^{-1} A P\right)^{[*]_{P^{*} H}}=P^{-1} A^{[*]_{H}} P .
$$

Moreover, with the help of (2.3), it is easily deduced that

$$
\left(P^{-1} A P\right)^{-1}=P^{-1} A^{-1} P
$$

where the inverses are understood in the sense of linear relations.

Thus, if $\mathrm{A}$ is a matrix, then changing the basis of $\mathbb{C}^{n}$ accordingly, we may always assume that $H$ and $A$ have the forms

$$
H={ }_{n-m}^{m}\left[\begin{array}{cc}
H_{1} & 0 \\
0 & 0
\end{array}\right] \text { and } A={ }_{n-m}^{m}\left[\begin{array}{cc}
m & n-m \\
A_{1} & A_{2} \\
A_{3} & A_{4}
\end{array}\right] \in \mathbb{C}^{n \times n}
$$

where $H_{1} \in \mathbb{C}^{m \times m}$ is nonsingular. When using these forms and identifying $A$ with the linear relation $A \subseteq \mathbb{C}^{2 n}$, then for the ease of simple notation we will usually omit the indication of dimensions of vectors if those are clear from the context. Thus, for example, we write

$$
A=\left\{\left(\begin{array}{c}
x_{1} \\
x_{2} \\
A_{1} x_{1}+A_{2} x_{2} \\
A_{3} x_{1}+A_{4} x_{2}
\end{array}\right): x_{1} \in \mathbb{C}^{m}, x_{2} \in \mathbb{C}^{n-m}\right\}=\left\{\left(\begin{array}{c}
x_{1} \\
x_{2} \\
A_{1} x_{1}+A_{2} x_{2} \\
A_{3} x_{1}+A_{4} x_{2}
\end{array}\right)\right\} .
$$

Proposition 2.6 Let $A \in \mathbb{C}^{n \times n}$ be a matrix. Then

$$
A^{[*]}=H^{-1} A^{*} H
$$


where $H^{-1}$ is the inverse in the sense of linear relations. In particular, if $H$ and $A$ have the forms as in (2.6) then

$$
A^{[*]}=\left\{\left(\begin{array}{c}
y_{1} \\
y_{2} \\
A_{1}^{[*]_{H}} y_{1} \\
z_{2}
\end{array}\right): A_{2}^{*} H_{1} y_{1}=0\right\} .
$$

Moreover, we have $\operatorname{dom} A^{[*]}=\mathbb{C}^{n}$ if and only if $A_{2}=0$.

Proof. We have

$$
H^{-1}=\left\{\left(\begin{array}{l}
x \\
z
\end{array}\right):\left(\begin{array}{c}
z \\
x
\end{array}\right) \in H\right\}=\left\{\left(\begin{array}{c}
H z \\
z
\end{array}\right)\right\}
$$

and, using Lemma 2.4,

$$
\begin{aligned}
H^{-1} A^{*} H & =H^{-1}\left\{\left(\begin{array}{c}
y \\
A^{*} H y
\end{array}\right)\right\}=H^{-1}\left\{\left(\begin{array}{c}
y \\
H z
\end{array}\right): H z=A^{*} H y\right\} \\
& =\left\{\left(\begin{array}{c}
y \\
z
\end{array}\right): H z=A^{*} H y\right\}=A^{[*]}
\end{aligned}
$$

and (2.7) is proved.

Let $H$ and $A$ be in the forms (2.6). By Lemma 2.4, we have that

$$
\begin{aligned}
A^{[*]} & =\left\{\left(\begin{array}{l}
y_{1} \\
y_{2} \\
z_{1} \\
z_{2}
\end{array}\right): A^{*} H\left(\begin{array}{l}
y_{1} \\
y_{2}
\end{array}\right)=H\left(\begin{array}{l}
z_{1} \\
z_{2}
\end{array}\right)\right\} \\
& =\left\{\left(\begin{array}{l}
y_{1} \\
y_{2} \\
z_{1} \\
z_{2}
\end{array}\right): A_{1}^{*} H_{1} y_{1}=H_{1} z_{1} \text { and } A_{2}^{*} H_{1} y_{1}=0\right\} .
\end{aligned}
$$

Since $H_{1}^{-1} A_{1}^{*} H_{1} y_{1}=A_{1}^{[*]_{H_{1}}} y_{1}$, we obtain (2.8) and since $H_{1}$ is invertible, we have $\operatorname{dom} A^{[*]}=$ $\mathbb{C}^{n}$ if and only if $A_{2}=0$.

\section{$3 \quad H$-symmetric and $H$-isometric matrices}

In the nondegenerate case, $H$-selfadjoint matrices are defined as matrices $A$ satisfying $A=A^{[*]}$. When generalizing this concept to the degenerate case, however, we have to take into account that for any $A \in \mathbb{C}^{n \times n}$, the relation $A^{[*]}$ is never a matrix when $H$ is singular. Thus, matrices satisfying $A=A^{[*]}$ do not exist. Instead, it is natural to consider matrices that are $H$-symmetric relations in the following sense. 
Definition 3.1 A linear relation $A$ in $\mathbb{C}^{n}$ is called $H$-symmetric if $A \subseteq A^{[*]}$.

We mention that $H$-symmetric relations in degenerate inner product spaces have been introduced in [22].

Example 3.2 In Example 1.1 we have that $M_{1}$ is $H$-symmetric.

Clearly, $A$ is $H$-symmetric if and only if $P^{-1} A P$ is $P^{*} H P$-symmetric for any invertible $P \in \mathbb{C}^{n \times n}$, cf. (2.3). At first sight, Definition 3.1 in the case of a matrix $A \in \mathbb{C}^{n \times n}$ may look a little bit weird, but the following proposition shows that this definition does make sense, because we will show that $H$-symmetry is equivalent to the condition $A^{*} H=H A$ which has been used as the definition for $H$-selfadjoint matrices in degenerate inner products in various sources.

Proposition 3.3 Let $A \in \mathbb{C}^{n \times n}$ be a matrix. Then the following statements are equivalent.

i) $A$ is $H$-symmetric, i.e., $A \subseteq A^{[*]}$.

ii) $A^{*} H=H A$.

If one of the conditions is satisfied, then ker $H$ is $A$-invariant. In particular, if $H$ and $A$ have the forms as in (2.6) then $A$ is $H$-symmetric if and only if $A_{1}$ is $H_{1}$-selfadjoint and $A_{2}=0$.

Proof. Let $\left(\begin{array}{c}y \\ A y\end{array}\right) \in A$. Then, by Lemma 2.4, we have

$$
\left(\begin{array}{c}
y \\
A y
\end{array}\right) \in A^{[*]} \Longleftrightarrow A^{*} H y=H A y
$$

This shows the equivalence of the two statements in Proposition 3.3. (For the implication i) $\Rightarrow i i$ ) observe that $A \subseteq A^{[*]}$ implies that the domain of $A^{[*]}$ is $\mathbb{C}^{n}$.)

For the remainder of the proof, let $H$ and $A$ be in the forms as in (2.6) and assume that $A$ is $H$-symmetric. Then using that the domain of $A^{[*]}$ is $\mathbb{C}^{n}$, we obtain from (2.8) that $A_{1} y_{1}+A_{2} y_{2}=A_{1}^{[*]_{H_{1}}} y_{1}$ and $A_{2}^{*} H_{1} y_{1}=0$ for all $y_{1} \in \mathbb{C}^{m}$. Since $H_{1}$ is invertible, this implies $A_{2}=0$ and we also obtain $A_{1}=A_{1}^{[*]_{H_{1}}}$, i.e., $A_{1}$ is $H_{1}$-selfadjoint.

Thus, a matrix is $H$-symmetric (in the sense of linear relations) if and only if it is $H$ selfadjoint (in the sense of matrices). At first sight, it may look a little bit disappointing that only one inclusion of the usual concept of "selfadjointness" (in the sense of linear relations) is fulfilled. However, this changes if we adjoin the inclusion $A \subseteq A^{[*]}$ once more, as the following proposition shows.

Proposition 3.4 Let $A \in \mathbb{C}^{n \times n}$ be a matrix. Then $A$ is $H$-symmetric if and only if

$$
A^{[*]}=\left(A^{[*]}\right)^{[*]} \text {. }
$$


Proof. If $A^{[*]}=\left(A^{[*]}\right)^{[*]}$ then, by Proposition 2.2 (iv), $A$ is $H$-symmetric. For the converse assume $A \subseteq A^{[*]}$. Then Proposition 2.2 (i) implies $\left(A^{[*]}\right)^{[*]} \subseteq A^{[*]}$. For the other inclusion observe that $A \subseteq A^{[*]}$ and Proposition 2.2 (iv) give

$$
\operatorname{dom} A^{[*]}=\operatorname{dom}\left(A^{[*]}\right)^{[*]}=\mathbb{C}^{n} .
$$

Moreover, Proposition 2.2 (ii) and Lemma 2.3 together with Proposition 3.3 imply

$$
\operatorname{mul} A^{[*]}=\operatorname{mul}\left(A^{[*]}\right)^{[*]}=\operatorname{ker} H .
$$

Hence, $A^{[*]}=\left(A^{[*]}\right)^{[*]}$.

Proposition 2.2 (iv) together with Proposition 3.4 imply the following corollary.

Corollary 3.5 Let $A \in \mathbb{C}^{n \times n}$ be a matrix. Then $A$ is $H$-symmetric if and only if

$$
A^{[*]}=A+(\operatorname{ker} H \times \operatorname{ker} H)
$$

Similar to $H$-symmetric matrices, $H$-isometric matrices can be defined by passing to the concept of linear relations. (We mention that $H$-isometric relations in degenerate inner product spaces have been introduced in [22].)

Definition 3.6 A linear relation $U$ in $\mathbb{C}^{n}$ is called $H$-isometric if $U^{-1} \subseteq U^{[*]}$.

We note that in the definition above $U^{-1}$ is the inverse in the sense of linear relations. E.g., if $H=0$, then every matrix is $H$-isometric. It follows from (2.4) and (2.5) that $U$ is $H$-isometric if and only if $P^{-1} U P$ is $P^{*} H P$-isometric for any invertible $P \in \mathbb{C}^{n \times n}$.

Proposition 3.7 Let $U \in \mathbb{C}^{n \times n}$ be a matrix. Then the following statements are equivalent.

i) $U$ is $H$-isometric, i.e., $U^{-1} \subseteq U^{[*]}$.

ii) $U^{*} H U=H$.

If one of the conditions is satisfied, then ker $H$ is $U$-invariant. In particular, if $H$ and $U$ have the forms as in (2.6), i.e.,

$$
U=\left(\begin{array}{cc}
U_{1} & U_{2} \\
U_{3} & U_{4}
\end{array}\right), \quad H=\left(\begin{array}{cc}
H_{1} & 0 \\
0 & 0
\end{array}\right)
$$

where $H_{1}$ is invertible, then $U$ is $H$-isometric if and only if $U_{1}$ is $H_{1}$-unitary and $U_{2}=0$. Moreover, we have

$$
(\operatorname{ran} U)^{[\perp]}=\operatorname{ker} H
$$


Proof. By Lemma 2.4, we have

$$
U^{-1} \subseteq U^{[*]} \Longleftrightarrow\left(\begin{array}{c}
U y \\
y
\end{array}\right) \in U^{[*]} \text { for all } y \in \mathbb{C}^{n} \Longleftrightarrow U^{*} H U y=H y \text { for all } y \in \mathbb{C}^{n}
$$

This shows the equivalence of the two statements in Proposition 3.7.

For the remainder of the proof, let $H$ and $U$ be in the forms as in (3.1) and assume that $U$ is $H$-isometric. Then we obtain from the identity $U^{*} H U=H$ that

$$
\left(\begin{array}{cc}
U_{1}^{*} H_{1} U_{1} & U_{1}^{*} H_{1} U_{2} \\
U_{2}^{*} H_{1} U_{1} & U_{2}^{*} H_{1} U_{2}
\end{array}\right)=\left(\begin{array}{cc}
H_{1} & 0 \\
0 & 0
\end{array}\right)
$$

This immediately implies $U_{1}^{*} H_{1} U_{1}=H_{1}$, i.e., $U_{1}$ is $H_{1}$-unitary. In particular, with $H_{1}$ also $U_{1}$ must be invertible. This finally yields $U_{2}=0$ and (3.2).

If we adjoin the inclusion $U^{-1} \subseteq U^{[*]}$ once more we get, similar to Proposition 3.4, the following characterization of $H$-isometric matrices.

Proposition 3.8 Let $U \in \mathbb{C}^{n \times n}$ be a matrix. Then $U$ is $H$-isometric if and only if

$$
\left(U^{-1}\right)^{[*]}=\left(U^{[*]}\right)^{[*]}
$$

Proof. Let $U$ be $H$-isometric, i.e., $U^{-1} \subseteq U^{[*]}$. Then, by Proposition 2.2 (i), we have $\left(U^{[*]}\right)^{[*]} \subseteq\left(U^{-1}\right)^{[*]}$. For the other inclusion, observe that, by Proposition 2.2 (iv), we have

$$
\operatorname{dom}\left(U^{[*]}\right)^{[*]}=\mathbb{C}^{n} .
$$

Thus, using $\left(U^{[*]}\right)^{[*]} \subseteq\left(U^{-1}\right)^{[*]}$, we obtain that

$$
\operatorname{dom}\left(U^{[*]}\right)^{[*]}=\operatorname{dom}\left(U^{-1}\right)^{[*]}=\mathbb{C}^{n} .
$$

Proposition 2.2 (ii) and (3.2) imply

$$
\operatorname{mul}\left(U^{-1}\right)^{[*]}=\left(\operatorname{dom} U^{-1}\right)^{[\perp]}=(\operatorname{ran} U)^{[\perp]}=\operatorname{ker} H
$$

and, with Lemma 2.3 and the $U$-invariance of ker $H$ (Proposition 3.7), we conclude

$$
\operatorname{mul}\left(U^{[*]}\right)^{[*]}=\operatorname{ker} H=\operatorname{mul}\left(U^{-1}\right)^{[*]} .
$$

Hence $\left(U^{-1}\right)^{[*]}=\left(U^{[*]}\right)^{[*]}$.

\section{$4 \quad H$-normal matrices}

Recall that in the case of invertible $H$, a matrix $A$ is called $H$-normal if and only if $A A^{[*]}=A^{[*]} A$. For the case that $H$ is singular and that $H$ and $A$ are given in the forms as in (2.6), a straightforward computation reveals

$$
A A^{[*]}=\left\{\left(\begin{array}{c}
y_{1} \\
y_{2} \\
A_{1} A_{1}^{[*]} y_{1}+A_{2} z_{2} \\
A_{3} A_{1}^{[*]} y_{1}+A_{4} z_{2}
\end{array}\right): A_{2}^{*} H_{1} y_{1}=0\right\}
$$


and

$$
A^{[*]} A=\left\{\left(\begin{array}{c}
y_{1} \\
y_{2} \\
A_{1}^{[*]} A_{1} y_{1}+A_{1}^{[*]} A_{2} y_{2} \\
z_{2}
\end{array}\right): A_{2}^{*} H_{1} A_{1} y_{1}+A_{2}^{*} H_{1} A_{2} y_{2}=0\right\} .
$$

However, even in the case that $A$ is $H$-symmetric (i.e., in the identities (4.1) and (4.2) we have $A_{1} A_{1}^{[*]}=A_{1}^{[*]} A_{1}=A_{1}^{2}$ and $A_{2}=0$ ), we only obtain the inclusion $A A^{[*]} \subseteq A^{[*]} A$, while the other inclusion $A^{[*]} A \subseteq A A^{[*]}$ is only satisfied if $A_{4}$ is invertible. This motivates the following definition.

Definition 4.1 $A$ relation $A$ in $\mathbb{C}^{n}$ is called $H$-normal if $A A^{[*]} \subseteq A^{[*]} A$.

As for the case of $H$-isometric and $H$-symmetric matrices, we obtain that the kernel of $H$ is always an invariant subspace for $H$-normal matrices.

Proposition 4.2 Let $A \in \mathbb{C}^{n \times n}$ be an $H$-normal matrix. Then ker $H$ is $A$-invariant. In particular, if $A$ and $H$ are in the forms as in (2.6), then $A$ is $H$-normal if and only if $A_{1}$ is $H_{1}$-normal and $A_{2}=0$.

Proof. Without loss of generality, we may assume that $A$ and $H$ are in the forms as in (2.6). Clearly, if $A_{1}$ is $H_{1}$-normal and $A_{2}=0$ then it follows directly from (4.1) and (4.2) that $A A^{[*]} \subseteq A^{[*]} A$, i.e., $A$ is $H$-normal. For the converse, assume that $A$ is $H$-normal. Then $A A^{[*]} \subseteq A^{[*]} A$ implies in particular that

$$
\left(A A^{[*]} \cap\left(\{0\} \times \mathbb{C}^{n}\right)\right) \subseteq\left(A^{[*]} A \cap\left(\{0\} \times \mathbb{C}^{n}\right)\right)
$$

Comparing the third block components of (4.1) and (4.2) this reduces to $A_{2} z=0$ for all $z_{2} \in \mathbb{C}^{n-m}$ and this is only possible if $A_{2}=0$. But then $A A^{[*]} \subseteq A^{[*]} A$ implies $A_{1} A_{1}^{[*]} y_{1}=A_{1}^{[*]} A_{1} y_{1}$ for all $y_{1} \in \mathbb{C}^{m}$ and we obtain that $A_{1}$ is $H_{1}$-normal. Clearly, ker $H$ is $A$-invariant, because of $A_{2}=0$. This concludes the proof.

With Propositions 3.3 and 3.7 we immediately obtain the following corollary.

Corollary $4.3 H$-symmetric and $H$-isometric matrices are $H$-normal.

The question arises, if we obtain a different characterization of $H$-normality in the style of Propositions 3.4 and 3.8 by the identity

$$
A^{[*]}\left(A^{[*]}\right)^{[*]}=\left(A^{[*]}\right)^{[*]} A^{[*]} .
$$

Let us investigate this question in detail. Without loss of generality assume that the matrix $A \in \mathbb{C}^{n \times n}$ and $H$ are given in the forms (2.6). Then using Proposition 2.2 (iv), we obtain

$$
\left(A^{[*]}\right)^{[*]}=\left\{\left(\begin{array}{c}
x_{1} \\
x_{2} \\
A_{1} x_{1}+A_{2} x_{2} \\
A_{3} x_{1}+A_{4} x_{2}
\end{array}\right)+\left(\begin{array}{c}
0 \\
w_{2} \\
0 \\
z_{2}
\end{array}\right)\right\}=\left\{\left(\begin{array}{c}
x_{1} \\
w_{2} \\
A_{1} x_{1}+A_{2} x_{2} \\
z_{2}
\end{array}\right)\right\} .
$$


Together with (2.8) this implies

$$
A^{[*]}\left(A^{[*]}\right)^{[*]}=\left\{\left(\begin{array}{c}
x_{1} \\
w_{2} \\
A_{1}^{[*]} A_{1} x_{1}+A_{1}^{[*]} A_{2} x_{2} \\
z_{2}
\end{array}\right): A_{2}^{*} H_{1}\left(A_{1} x_{1}+A_{2} x_{2}\right)=0\right\}
$$

and

$$
\left(A^{[*]}\right)^{[*]} A^{[*]}=\left\{\left(\begin{array}{c}
y_{1} \\
y_{2} \\
A_{1} A_{1}^{[*]} y_{1}+A_{2} x_{2} \\
z_{2}
\end{array}\right): A_{2}^{*} H_{1} y_{1}=0\right\} .
$$

With the help of these formulas, we obtain that (4.3) may be satisfied even if the matrix $A \in \mathbb{C}^{n \times n}$ is not $H$-normal, see Example 4.4 below.

Example 4.4 Let $A$ and $H$ be given as

$$
A=\left(\begin{array}{c|c}
A_{1} & A_{2} \\
\hline A_{3} & A_{4}
\end{array}\right):=\left(\begin{array}{cc|c}
1 & 0 & 1 \\
0 & 1 & 0 \\
\hline 0 & 0 & 0
\end{array}\right), \quad H=\left(\begin{array}{c|c}
H_{1} & 0 \\
\hline 0 & 0
\end{array}\right):=\left(\begin{array}{ll|l}
0 & 1 & 0 \\
1 & 0 & 0 \\
\hline 0 & 0 & 0
\end{array}\right) .
$$

Then $A$ is not $H$-normal, because ker $H$ is not $A$-invariant. However, from (4.4) and (4.5), we immediately obtain

$$
A^{[*]}\left(A^{[*]}\right)^{[*]}=\left\{\left(\begin{array}{c}
x_{1} \\
w_{2} \\
x_{1}+A_{2} x_{2} \\
z_{2}
\end{array}\right): A_{2}^{*} H_{1} x_{1}=0\right\}=\left(A^{[*]}\right)^{[*]} A^{[*]} .
$$

The following result shows that the set of matrices satisfying (4.3) contains the set of $H$-normal matrices.

Proposition 4.5 Let $A \in \mathbb{C}^{n \times n}$ be a matrix. If $A$ is $H$-normal, then

$$
A^{[*]}\left(A^{[*]}\right)^{[*]}=\left(A^{[*]}\right)^{[*]} A^{[*]} \text {. }
$$

Proof. Without loss of generality let $A$ and $H$ be in the forms (2.6). Then we obtain by Proposition 4.2 that $A_{2}=0$. The identities (4.4) and (4.5) imply

$$
\left(A^{[*]}\right)^{[*]} A^{[*]}=\left\{\left(\begin{array}{c}
y_{1} \\
y_{2} \\
A_{1} A_{1}^{[*]} y_{1} \\
w_{2}
\end{array}\right)\right\} \quad \text { and } \quad A^{[*]}\left(A^{[*]}\right)^{[*]}=\left\{\left(\begin{array}{c}
x_{1} \\
x_{2} \\
A_{1}^{[*]} A_{1} x_{1} \\
z_{2}
\end{array}\right)\right\} .
$$

Indeed, these two sets are equal because of the $H_{1}$-normality of $A_{1}$ which is guaranteed by Proposition 4.2.

Next, let us compare $H$-normal matrices with Moore-Penrose $H$-normal matrices. We obtain the following result. 
Proposition 4.6 Let $A \in \mathbb{C}^{n \times n}$ be a matrix. Then the following statements are equivalent.

i) $A$ is $H$-normal, i.e., $A A^{[*]} \subseteq A^{[*]} A$.

ii) $A$ is Moore-Penrose $H$-normal and $A^{[*]}\left(A^{[*]}\right)^{[*]}=\left(A^{[*]}\right)^{[*]} A^{[*]}$.

Proof. Without loss of generality, let $A$ and $H$ have the forms (2.6). Then the MoorePenrose generalized inverse of $H$ is given by

$$
H^{\dagger}=\left(\begin{array}{cc}
H_{1}^{-1} & 0 \\
0 & 0
\end{array}\right)
$$

and the matrix $A$ is Moore-Penrose $H$-normal if and only if

$$
\left(\begin{array}{cc}
A_{1}^{*} H_{1} A_{1} & A_{1}^{*} H_{1} A_{2} \\
A_{2}^{*} H_{1} A_{1} & A_{2}^{*} H_{1} A_{2}
\end{array}\right)=\left(\begin{array}{cc}
H_{1} A_{1} H_{1}^{-1} A_{1}^{*} H_{1} & 0 \\
0 & 0
\end{array}\right)
$$

" $i) \Rightarrow i i)$ ": If $A$ is $H$-normal, then by Proposition 4.2 we have that $A_{1}$ is $H_{1}$-normal and that $A_{2}=0$. Then (4.6) is satisfied and the remainder follows from Proposition 4.5.

"ii $\Rightarrow$ i)": Let $A$ be Moore-Penrose $H$-normal and let $A^{[*]}\left(A^{[*]}\right)^{[*]}=\left(A^{[*]}\right)^{[*]} A^{[*]}$. Then by (4.6) we have $A_{2}^{*} H_{1} A_{1}=0$ and $A_{2}^{*} H_{1} A_{2}=0$. Comparing this with (4.4), we find that $\operatorname{dom} A^{[*]}\left(A^{[*]}\right)^{[*]}=\mathbb{C}^{n}$. But then, we must have $\operatorname{dom}\left(A^{[*]}\right)^{[*]} A^{[*]}=\mathbb{C}^{n}$ as well which, using (4.5), implies $A_{2}^{*} H_{1} y_{1}=0$ for all $y_{1} \in \mathbb{C}^{m}$. From this, we obtain $A_{2}=0$. But then $A^{[*]}\left(A^{[*]}\right)^{[*]}=\left(A^{[*]}\right)^{[*]} A^{[*]}$ reduces to $A_{1}^{[*]} A_{1} x_{1}=A_{1} A_{1}^{[*]} x_{1}$ for all $x_{1} \in \mathbb{C}^{m}$ which implies $H_{1}$-normality of $A_{1}$. From this and Proposition 4.2, we finally obtain that $A$ is $H$-normal.

As a consequence, we obtain that the set of $H$-normal matrices is a strict subset of the set of Moore-Penrose $H$-normal matrices, because it has been shown in [17, Example 6.1] that there exist Moore-Penrose $H$-normal matrices $A$ such that ker $H$ is not $A$-invariant.

The fact that the kernel of $H$ is invariant for $H$-normal matrices allows the generalization of extension results for $H$-semidefinite invariant subspaces of normal matrices for invertible $H$ to the case of singular $H$. For example, if $A$ is $H$-normal and $H$ is invertible, then any $H$-nonnegative subspace that is invariant for both $A$ and $A^{[*]}$ can be extended to an $A$-invariant maximal $H$-nonnegative subspace, see [15]. This result now easily generalizes to the case of singular $H$. Here, an invariant subspace $\mathcal{U} \subseteq \mathbb{C}^{n}$ of a linear relation $A$ in $\mathbb{C}^{n}$ is defined by the implication

$$
x \in \mathcal{U} \text { and }\left(\begin{array}{l}
x \\
y
\end{array}\right) \in A \quad \Longrightarrow \quad y \in \mathcal{U}
$$

Theorem 4.7 Let $A \in \mathbb{C}^{n \times n}$ be $H$-normal, and let $\mathcal{M}_{0}$ be an $H$-nonnegative $A$-invariant subspace that is also invariant for $A^{[*]}$. Then there exists an A-invariant maximal $H$ nonnegative subspace $\mathcal{M}$ containing $\mathcal{M}_{0}$ that is also invariant for $A^{[*]}$. 
Proof. Without loss of generality assume that $A$ and $H$ are in the forms (2.6) and that $\mathcal{M}_{0}$ can be written as a direct sum $\mathcal{M}_{0}=\mathcal{M}_{1}+\mathcal{M}_{2}$, where $\mathcal{M}_{2} \subseteq$ ker $H$ and

$$
\mathcal{M}_{1}=\left\{\left(\begin{array}{c}
x_{1} \\
0
\end{array}\right): x_{1} \in \widetilde{\mathcal{M}}_{1}\right\}
$$

for some subspace $\widetilde{\mathcal{M}}_{1} \subseteq \mathbb{C}^{m}$. It is easy to verify that the $A$ - and $A^{[*]}$-invariance of $\mathcal{M}_{0}$ imply that $\widetilde{\mathcal{M}}_{1}$ is $A_{1}$-invariant as well as $A_{1}^{[*]}$-invariant. Thus, by [15] there exists an $A_{1}$ invariant maximal $H$-nonnegative subspace $\mathcal{M}_{\max }$ that is also invariant for $A_{1}^{[*]}$. Setting $\mathcal{M}=\mathcal{M}_{\text {max }} \dot{+} \operatorname{ker} H$, we obtain that $\mathcal{M}$ contains $\mathcal{M}_{0}$ and is $A$-invariant and maximal $H$-nonnegative. It is easy to check that $\mathcal{M}$ is also $A^{[*]}$-invariant.

If we drop the assumption that $\mathcal{M}_{0}$ is $A^{[*]}$-invariant, then extension results are not as immediate. Indeed, it has been shown in [18] that there exist $H$-normal matrices (in the case of invertible $H$ ) that have an invariant $H$-nonnegative subspace that cannot be extended to an invariant maximal $H$-nonnegative subspace. (There still exist such counterexamples if one restricts the subspace to be $H$-positive rather than $H$-nonnegative.) Thus, stronger conditions have to be imposed on an $H$-normal matrix such that extension of semidefinite invariant subspaces can be guaranteed, see [18, 19].

We conclude the paper by generalizing a result concerning the extension of $H$-positive invariant subspaces of $H$-normal matrices obtained in [18] to the case of singular $H$. The fact that ker $H$ is always an invariant subspace for $H$-normal matrices plays a key role in this proof.

Theorem 4.8 Let $A \in \mathbb{C}^{n \times n}$ be $H$-normal, and let $\mathcal{M}_{0}$ be an $H$-positive $A$-invariant subspace. Let $\mathcal{M}_{\text {com }}$ be a direct complement of ker $H$ in $\mathcal{M}_{0}^{[\perp]}$, that is, $\mathcal{M}_{0}^{[\perp]}=\mathcal{M}_{\text {com }} \dot{+} \operatorname{ker} H$. Define

$$
A_{22}:=\left.P X\right|_{\mathcal{M}_{\text {com }}}: \mathcal{M}_{\text {com }} \rightarrow \mathcal{M}_{\text {com }},
$$

where $P$ is the projection onto $\mathcal{M}_{\text {com }}$ along $\mathcal{M}_{0} \dot{+}$ ker $H$. Then $\mathcal{M}_{\text {com }}$ is nondegenerate. Equip $\mathcal{M}_{\text {com }}$ with the indefinite inner product induced by $H$. Assume that

$$
\sigma\left(A_{22}+A_{22}^{[*]}\right) \subseteq \mathbb{R} \quad \text { or } \quad \sigma\left(A_{22}-A_{22}^{[*]}\right) \subseteq i \mathbb{R} .
$$

Then there exists an $A$-invariant maximal $H$-nonnegative subspace $\mathcal{M}$ that contains $\mathcal{M}_{0}$ and that is also $A^{[*]}$-invariant.

The condition (4.7) is independent of the particular choice of a direct complement $\mathcal{M}_{\text {com }}$ of ker $H$ in $\mathcal{M}_{0}^{[\perp]}$.

Proof. Since $\mathcal{M}_{0}$ is $H$-positive, we have that $\mathcal{M}_{0}^{[\perp]}$ is a direct complement of $\mathcal{M}_{0}$. Moreover, it is clear that a complement of ker $H$ is nondegenerate. (By default, i.e., nonexistence of $H$-neutral vectors, $\{0\}$ is a nondegenerate subspace.) Thus, without loss of generality, we may assume that $\mathcal{M}_{0}=\operatorname{span}\left(e_{1}, \ldots, e_{k}\right)$ and $\mathcal{M}_{\text {com }}=\operatorname{span}\left(e_{k+1}, \ldots, e_{k+l}\right), l \geq 0$, and 
$\mathcal{M}_{0}^{[\perp]}=\operatorname{span}\left(e_{k+1}, \ldots, e_{n}\right)$, where $e_{j}$ denotes the $j$-th unit vector. Then $A$ and $H$ have the corresponding block forms

$$
A=\left(\begin{array}{ccc}
A_{11} & A_{12} & A_{13} \\
0 & A_{22} & A_{23} \\
0 & A_{32} & A_{33}
\end{array}\right), \quad H=\left(\begin{array}{ccc}
I_{k} & 0 & 0 \\
0 & H_{22} & 0 \\
0 & 0 & 0
\end{array}\right)
$$

where $H_{22}$ is invertible. In this representation of $A$, we have

$$
A_{1}=\left(\begin{array}{cc}
A_{11} & A_{12} \\
0 & A_{22}
\end{array}\right), \quad \text { and } \quad H_{1}=\left(\begin{array}{cc}
I_{k} & 0 \\
0 & H_{22}
\end{array}\right) \text {, }
$$

where $A_{1}$ and $H_{1}$ are defined in analogy to the decomposition (2.6). By Proposition 4.2 we have that $A_{1}$ is $H_{1}$-normal and that ker $H$ is $A$-invariant which implies $A_{13}=0$ and $A_{23}=0$. Setting

$$
\widetilde{\mathcal{M}}_{0}=\operatorname{ran}\left(\begin{array}{c}
I_{k} \\
0
\end{array}\right) \subseteq \mathbb{C}^{k+l}
$$

which is an $A_{1}$-invariant $H_{1}$-positive subspace, we obtain, given the condition on $A_{22}$, that by $[18$, Theorem 4.4$]$ there exists an $A_{1}$-invariant maximal $H_{1}$-nonnegative subspace $\widehat{\mathcal{M}}_{0}$ of dimension $\nu_{+}(H)=\nu_{+}\left(H_{1}\right)$ containing $\widetilde{\mathcal{M}}_{0}$. We choose appropriate matrices $M_{11}, M_{12}$ and write $\widehat{\mathcal{M}}_{0}$ in the following way

$$
\widehat{\mathcal{M}}_{0}=\operatorname{ran}\left(\begin{array}{cc}
I_{k} & M_{11} \\
0 & M_{12}
\end{array}\right)
$$

Let

$$
\mathcal{M}:=\operatorname{ran}\left(\begin{array}{ccc}
I_{k} & M_{11} & 0 \\
0 & M_{12} & 0 \\
0 & 0 & I_{n-k-l}
\end{array}\right) .
$$

Then it is straightforward to check that $\mathcal{M}$ is an $A$-invariant, $H$-nonnegative subspace of dimension $\nu_{+}(H)+\nu_{0}(H)$ containing $\mathcal{M}_{0}$. Clearly, $\mathcal{M}$ is also invariant for $A^{[*]}$.

It remains to show that the condition (4.7) is independent of the particular choice of the direct complement $\mathcal{M}_{\text {com }}$. But choosing a different direct complement $\mathcal{M}_{\text {new }}$ of ker $H$ in $\mathcal{M}_{0}^{[\perp]}=\operatorname{span}\left(e_{k+1}, \ldots, e_{n}\right)$ amounts to a change of basis given by a matrix of the form

$$
S=\left(\begin{array}{ccc}
I_{k} & 0 & 0 \\
0 & S_{22} & 0 \\
0 & S_{32} & I_{n-k-l}
\end{array}\right)
$$

with $S_{22}$ invertible. With respect to the decomposition $\mathbb{C}^{n}=\mathcal{M}_{0} \dot{+} \mathcal{M}_{\text {new }} \dot{+}$ ker $H$ and the new basis, $A$ and $H$ take the forms

$$
\tilde{A}=S^{-1} A S=\left(\begin{array}{ccc}
A_{11} & * & 0 \\
0 & S_{22}^{-1} A_{22} S_{22} & 0 \\
0 & * & A_{33}
\end{array}\right), \tilde{H}=S^{*} H S=\left(\begin{array}{ccc}
I_{k} & 0 & 0 \\
0 & S_{22}^{*} H_{22} S_{22} & 0 \\
0 & 0 & 0
\end{array}\right) .
$$

The compressions of $\tilde{A}$ and $\tilde{H}$ to $\mathcal{M}_{\text {new }}$ are $S_{22}^{-1} A_{22} S_{22}$ and $S_{22}^{*} H_{22} S_{22}$. Clearly, condition (4.7) is satisfied for these compressions if and only if (4.7) is satisfied for $A_{22}$ and $H_{22}$. 


\section{Acknowledgment}

We thank Heinz Langer for asking a question that initiated this research and Peter Jonas for fruitful comments on an earlier version of this paper.

\section{References}

[1] G. Ammar, C. Mehl, and V. Mehrmann, Schur-like Forms for Matrix Lie Groups, Lie Algebras and Jordan Algebras. Linear Algebra Appl. 287 (1999), 11-39.

[2] T. Ya. Azizov, Completely Continuous Operators that are Selfadjoint with Respect to a Degenerate Indefinite Metric (Russian). Mat. Issled. 7 (1972), 237-240, 259.

[3] T. Ya. Azizov and I. S. Iohvidov, Linear Operators in Spaces with an Indefinite Metric. John Wiley and Sons, Ltd., Chichester, 1989. (Translated from Russian.)

[4] V. Bolotnikov, C. K. Li, P. Meade, C. Mehl, and L. Rodman, Shells of Matrices in Indefinite Inner Product Spaces. Electron. J. Linear Algebra 9 (2002), 67-92.

[5] R. Cross, Multivalued Linear Operators. Marcel Dekker Inc., 1998.

[6] A. Dijksma and H.S.V. de Snoo, Symmetric and Selfadjoint Relations in Krein Spaces I. Oper. Theory Adv. Appl. 24 (1987), 145-166.

[7] A. Dijksma and H.S.V. de Snoo, Symmetric and Selfadjoint Relations in Krein Spaces II. Ann. Acad. Sci. Fenn. Math. 12 (1987), 199-216.

[8] I. Gohberg, P. Lancaster, and L. Rodman, Matrices and Indefinite Scalar Products. Birkhäuser, 1983.

[9] I. Gohberg, P. Lancaster, and L. Rodman, Indefinite Linear Algebra. Birkhäuser, 2005.

[10] I. Gohberg and B. Reichstein, On Classification of Normal Matrices in an Indefinite Scalar Product. Integral Equations Operator Theory 13 (1990), 364-394.

[11] M. Kaltenbäck and H. Woracek, Selfadjoint Extensions of Symmetric operators in Degenerated Inner Product Spaces, Integral Equations Operator Theory 28 (1997), 289-320.

[12] P. Lancaster, A. S. Markus, and P. Zizler, The Order of Neutrality for Linear Operators on Inner Product Spaces. Linear Algebra Appl. 259 (1997), 25-29.

[13] P. Lancaster and L. Rodman, Algebraic Riccati Equations. Clarendon Press, 1995.

[14] H. Langer, R. Mennicken, and C. Tretter, A Self-Adjoint Linear Pencil $Q-\lambda P$ of Ordinary Differential Operators. Methods Funct. Anal. Topology 2 (1996), 38-54. 
[15] H. Langer, Invariante Teilräume definisierbarer J-selbstadjungierter Operatoren. Ann. Acad. Sci. Fenn. Ser. A.I. Math. 475 (1971), 1-23

[16] C. K. Li, N. K. Tsing, and F Uhlig, Numerical Ranges of an Operator on an Indefinite Inner Product Space. Electron. J. Linear Algebra 1 (1996), 1-17.

[17] C. Mehl, A. Ran, and L. Rodman, Semidefinite Invariant Subspaces: Degenerate Inner Products. Oper. Theory Adv. Appl. 149 (2004), 467-486.

[18] C. Mehl, A. Ran, and L. Rodman, Hyponormal Matrices and Semidefinite Invariant Subspaces in Indefinite Inner Products. Electron. J. Linear Algebra 11 (2004), 192204.

[19] C. Mehl, A. Ran, and L. Rodman, Extension to Maximal Semidefinite Invariant Subspaces for Hyponormal Matrices in Indefinite Inner Products. Linear Algebra Appl., to appear.

[20] C. Mehl and L. Rodman, Symmetric Matrices with Respect to Sesquilinear Forms. Linear Algebra Appl. 349 (2002), 55-75.

[21] V. Mehrmann, Existence, Uniqueness, and Stability of Solutions to Singular Linear Quadratic Optimal Control Problems. Linear Algebra Appl. 121 (1989), 291-331.

[22] B. C. Ritsner, The Theory of Linear Relations. (Russian) Voronezh, Dep. VINITI, No. 846-82, 1982.

[23] H. Woracek, Resolvent Matrices in Degenerated Inner Product Spaces. Math. Nachr. 213 (2000), 155-175. 